\title{
Coherent Anti-Stokes Raman Scattering Microscopy
}

\author{
Mamoru Hashimoto ${ }^{1}$ \\ ${ }^{1}$ Department of Systems and Human Science, Graduate School of Engineering Science, Osaka University
}

Received March 26, 2002; accepted March 29, 2002

In this paper, new multiphoton micros-
copy using CARS (coherent anti-Stokes
Raman scattering) spectroscopy is de-
scribed. CARS microscopy has the fea-
tures of non-staining molecular mapping
by molecular vibration imaging and three-
dimensional resolution by multiphoton

process. A picosecond tunable laser and suitable optical filters provide the CARS imaging in the fingerprint region, and multi-focus excitation using a rotatorymicrolens array enables the multi-spectral imaging.

Key words: Multiphoton microscopy, Raman spectroscopy, Nonlinear optics, CARS spectroscopy

Recently, new microscopes utilizing non-linear spectroscopy have been developed. The most widely used multiphoton microscopy is multiphoton excitation fluorescence microscopy [2]. However, multiphoton fluorescence microscopy is the same as conventional fluorescence microscopy in the point of fluorescence imaging with staining. To extract the analytical molecules and/or ions, proper staining is essential. The staining procedure requires technical skills and often damages the functions of the cells. Hence, stainingfree, realtime molecular imaging of the cell with submicrometer 3-dimensional resolution is strongly required.

Since a molecule consists of particles (atoms) and springs (chemical bonds), the molecule vibrates and these frequencies depend on the kinds of atoms and chemical bonds. Therefore, the molecular vibration is sensitive to the molecular species and conformations. The vibrational spectroscopy (infrared and Raman spectroscopy) is widely used to determine the molecular species in the analytical chemistry, but it is not applied to biological studies so much. The biological sample contains a lot of water that has strong absorption in the infrared region, and the spatial resolution of infrared spectroscopy is limited to a few tens of micrometer by the diffraction limit. The spontaneous emission of Raman scattering is very weak compared with the auto-fluorescence of the biological sample. We consider that the combination

Correspondence to: Mamoru Hashimoto, Department of Systems and Human Science, Graduate School of Engineering Science, Osaka University, Machikaneyama 1-3, Toyonaka, Osaka 560-8531, Japan. of non-linear Raman spectroscopy, coherent anti-Stokes Raman scattering (CARS) spectroscopy, and microscopy is suitable for the biological application of non-staining imaging.

Figure 1 shows the CARS process. CARS is a fourphoton process $[9,11]$. In CARS, two laser beams whose frequencies are $\omega_{1}$ and $\omega_{2}$ are mixed into the sample. When the frequency difference $\omega_{1}-\omega_{2}$ is equal to the frequency of the Raman active molecular vibration $\Omega$, CARS is generated as the coherent emission at $2 \omega_{1}-\omega_{2}$. The advantages of CARS spectroscopy over conventional Raman (spontaneous Raman) spectroscopy are the high intensity emission and the separation from undesirable fluorescence signal. The CARS emission is separable from the fluorescence, since the wavelength of CARS is shorter than that of excitation beams. The spectrometer is fundamentally unnecessary in CARS spectroscopy. CARS spectrum is observed to scan the frequency difference of the incident $\omega_{1}$ and $\omega_{2}$ beams; the typical wavelength of the $\omega_{1}$ beam is fixed and that of the $\omega_{2}$ beam is scanned.

The combination of CARS spectroscopy and microscopy was proposed and demonstrated by Duncan et al. [3] earlier than the development of multiphoton fluorescence microscopy [2]. In their experiments, however, the CARS excitation beams were not tightly focused, and the microscope objective was used to magnify the CARS image. The effect of the resolution enhancement by multiphoton excitation was not exhibited.

One of the advantages of multiphoton microscopy is 


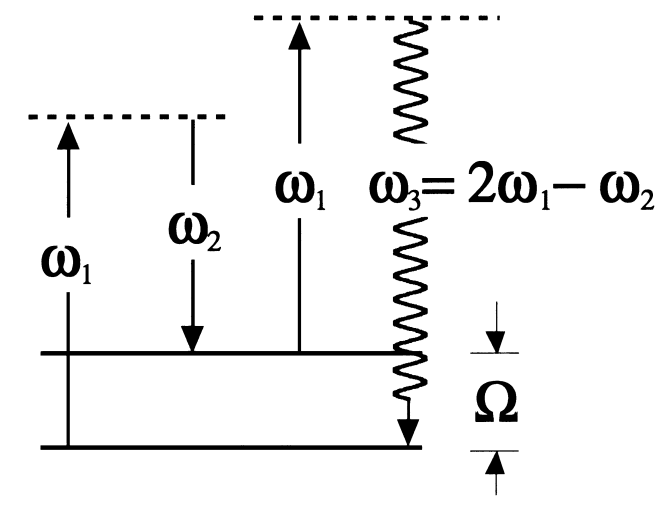

Fig. 1. CARS process.

the resolution enhancement. The excitation volume size is limited by the diffraction limit in single-photon excitation, but the non-linear effect overcomes the diffraction limit. We consider that if the excitation beams are tightly focused, CARS is generated around the focal point and the molecular information from the local volume will be obtained like multiphoton fluorescence microscopy. Because CARS is the coherent emission contrary to the fluorescence, we have to take account of the interference between the emissions generated from different points. This interference effect in the non-linear optics is known as the phase matching condition [10]. We derived the transfer function of CARS microscopy using the theory of coherent optics and estimated the three dimensional optical property. As the result, it was found that the phase matching condition is easily satisfied by tightly focusing, and the resolution of the CARS microscopy without the pinhole of the detector side is almost same as confocal fluorescence microscopy under the weak contrast assumption $[4,5]$.

Zumbusch et al. [14] constructed a tight focusing CARS microscopy system using a femtosecond laser and succeeded in observing the sectionized CARS images. They also applied it to the biological samples. We developed a CARS microscopy system for observing in the fingerprint region [6].

In the previous studies, CARS microscopy was realized in the higher Raman shift region $\left(>2000 \mathrm{~cm}^{-1}\right)$. The molecular vibrations of $\mathrm{CH}, \mathrm{OH}$, and $\mathrm{NH}$ stretching were observable in this region, but it was difficult to determine the molecular species from these vibrations, because any biological sample has these vibrations. We constructed the CARS microscopy system for lower Raman shift region (fingerprint region) by using a tunable picosecond laser.

Although molecular vibrations in the fingerprint region are more sensitive to molecular species and conformations than those in higher frequency region, the wavelengths of the CARS are close to those of the $\omega_{1}$ and $\omega_{2}$ beams. We used a filter stack made of 4 filters with different transmittance specifications, because a single filter is not enough to cut the $\omega_{1}$ and $\omega_{2}$ beams.

Since CARS is a nonlinear optical phenomenon, an ultra-fast laser with high peak intensity but low average power is suitable for the light source. However, femtosecond pulse is too fast to observe CARS, because the femtosecond pulse has a broad spectrum. The typical spectral bandwidth of femtosecond pulse is ten to twenty nanometer, which corresponds with 100 to $200 \mathrm{~cm}^{-1}$ at $800 \mathrm{~nm}$, though typical spectral bandwidth of Raman band is a few $\mathrm{cm}^{-1}$. The pulse duration and the spectral bandwidth are unable to be minimized simultaneously, because of the uncertainty principle. We used a picosecond laser to satisfy the high spectral resolution and the high efficiency of CARS.

Figure 2 shows the developed system. The $100 \mathrm{fs}$ pulse from a mode-locked Ti: Sapphire laser (fs Ti: $\mathrm{S}$ ) is amplified with a regeneration amplifier (ps RGA) and is converted to 2 ps pulse by restricting the spectral bandwidth. Resultant picosecond pulse is divided to two; one is used for $\omega_{1}$ beam and another for excitation of an optical parametric amplifier (ps OPA). An optical parametric amplifier generates the tunable picosecond pulse and it is used for $\omega_{2}$ beam. The spectral bandwidths of $\omega_{1}$ and $\omega_{2}$ beams are $0.85 \mathrm{~nm}(14.5$ $\left.\mathrm{cm}^{-1}\right)$ and $1.45 \mathrm{~nm}\left(21.5 \mathrm{~cm}^{-1}\right)$, respectively. The $\omega_{1}$ and $\omega_{2}$ beams are spatially superimposed on the beamsplitter and temporally overlapped with the optical delays, and then introduced to a transmission type optical microscope. In the microscope, two objectives are placed opposite each other. Two beams are tightly focused with the first objective, and CARS emission is collected with another one. The collected CARS emission is detected with an avalanche photodiode (APD) through the filter stack to remove the excitation beams. The image is obtained by scanning the sample with an xyz piezo-electric stage (PZT). The spectral resolution of the system is about $30 \mathrm{~cm}^{-1}$, and the spatial resolution is smaller than $1 \mu \mathrm{m}$ in the lateral direction and $3.2 \mu \mathrm{m}$ in the depth direction with NA=0.65 objectives [6].

Figure 3 shows the CARS images of polystyrene beads with $1 \mu \mathrm{m}$ diameter at the Raman shifts of $1030 \mathrm{~cm}^{-1}$ (a) and $980 \mathrm{~cm}^{-1}$ (b). It was found that the intensity of Fig. 3 (b) is higher than that of (a). The lateral resolution is smaller than $1 \mu \mathrm{m}$ because the $1 \mu \mathrm{m}$ beads are clearly observed. Figure 4 shows the CARS image of a yeast cell at $1215 \mathrm{~cm}^{-1}$. This

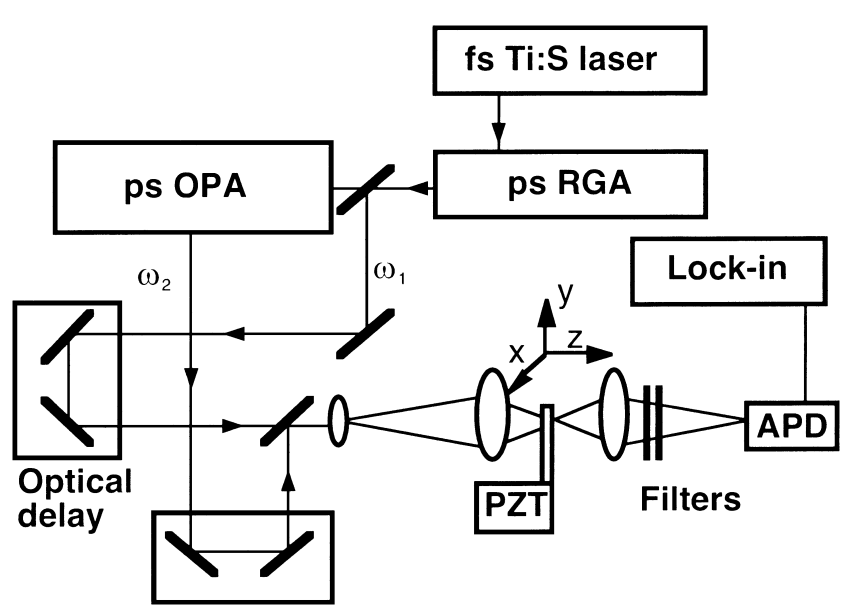

Fig. 2. The layout of CARS microscopy system. 

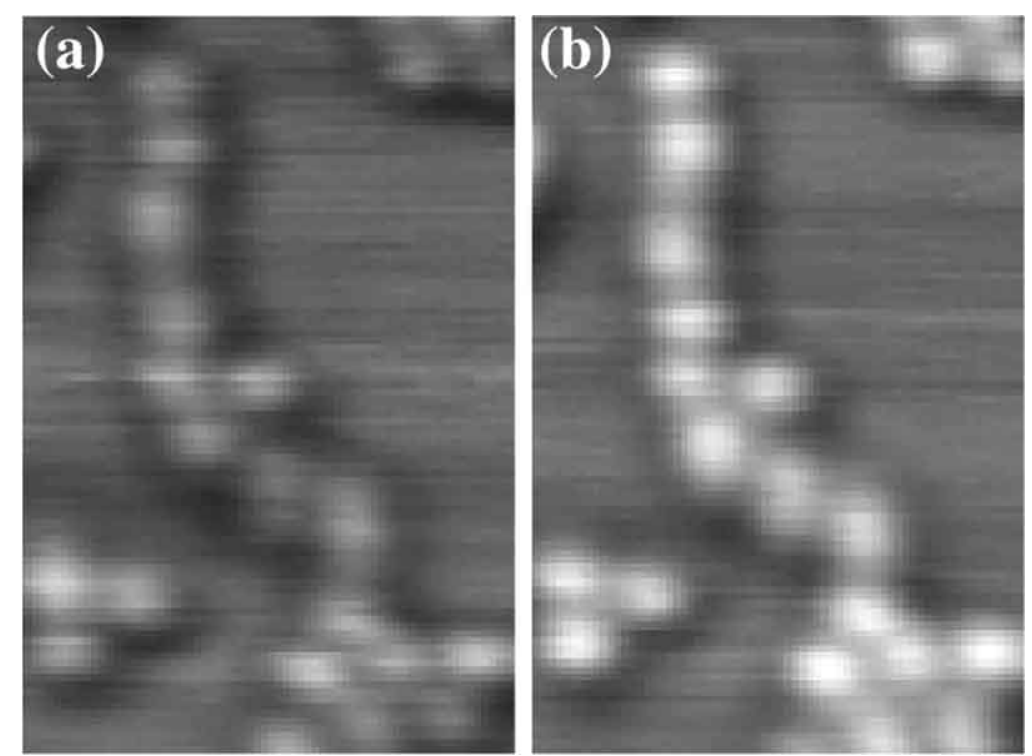

Fig. 3. CARS image of polystyrene beams with $1 \mu \mathrm{m}$ diameter at $1030 \mathrm{~cm}^{-1}$ (a) and $980 \mathrm{~cm}^{-1}(\mathbf{b})$.

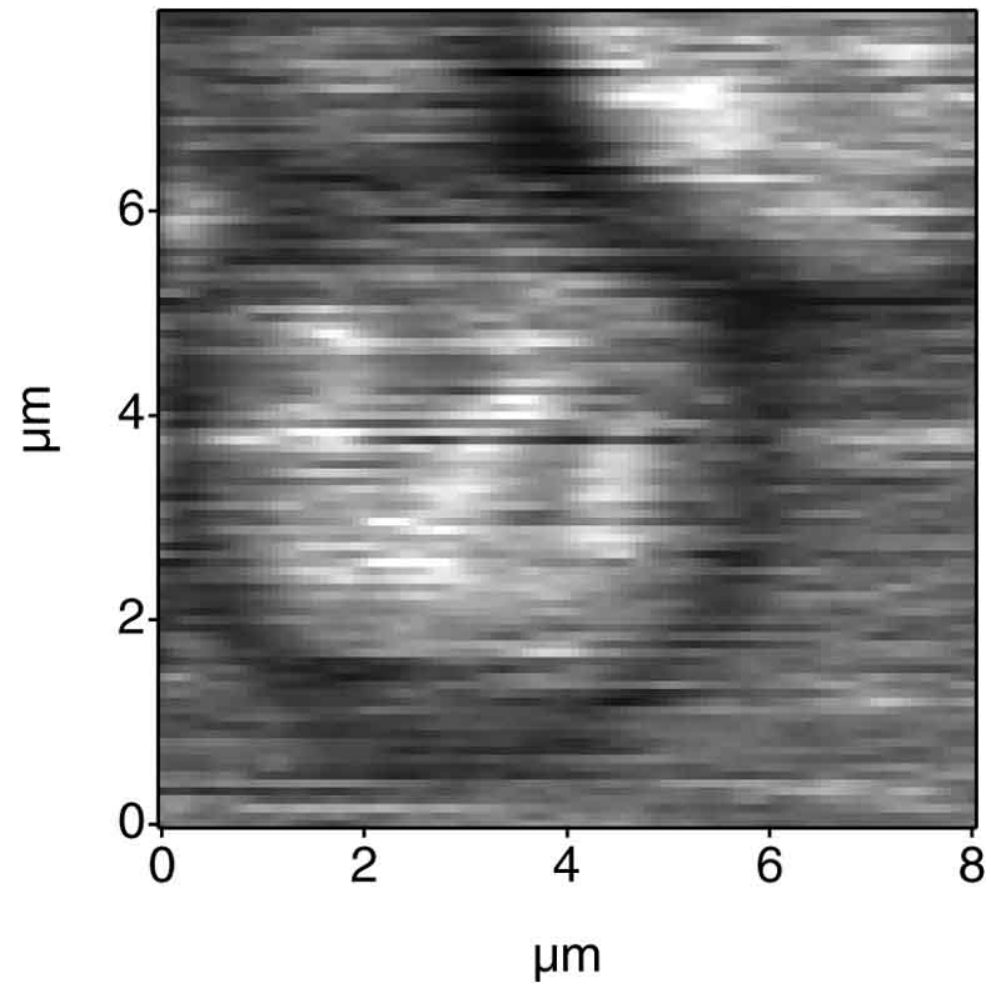

Fig. 4. CARS image of yeast cell at $1215 \mathrm{~cm}^{-1}$.

Raman shift corresponds to the amido III of protein.

A lot of images with different Raman shifts are required to obtain the molecular mapped image in CARS microscopy in order to identify the molecular species. It required long experimental time to take one image when used the stage scanning method. We have constructed an attractive system for fast imaging using a rotating microlens array. A microlens array is the gathering of small lenses $[8$,
12]. When the parallel beam is inputted to the microlens array, many spots are generated and thus, the sample is excited at these spots simultaneously. The CARS generated at these spots are collected with another side of the objective and are observed with a two-dimensional detector. By rotating the microlens array, these spots scan on a plane and the sectionized CARS image is obtained simultaneously. We have constructed a multi-focus CARS microscopy system 


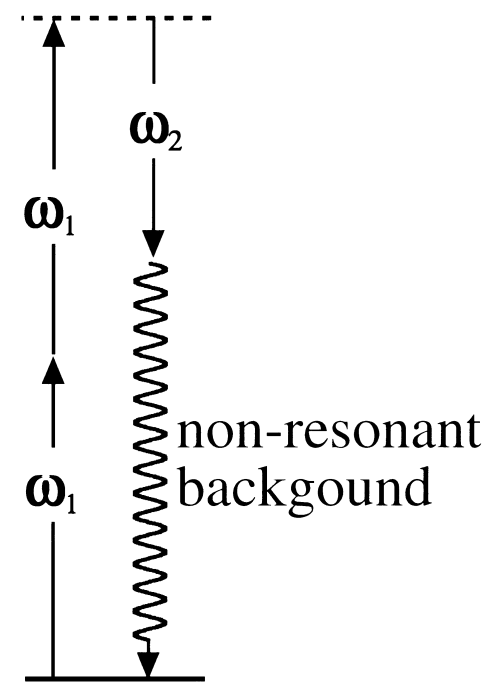

Fig. 5. The diagram of the non-resonant background process.

using a picosecond tunable laser system shown in Fig. 2. The parallel excitation and detection enabled the fast scanning, and the observation time was reduced to about 1/100 [7].

The present quality of CARS imaging is not satisfactory for the biological application because it still results in low contrast due to non-resonant background and poor spectral resolution. Figure 5 shows the diagram of the non-resonant background process. The non-resonant background is generated with CARS, and it has not any spectral feature. The spectral resolution of the present system is about $30 \mathrm{~cm}^{-1}$ which is larger than the typical Raman band of a few $\mathrm{cm}^{-1}$. This background component and poor spectral resolution reduce the contrast of the CARS image. Recently, epi-detection [13] and polarization control [1] were proposed to remove the non-resonant background. The direction of CARS emission depends on the size of sample by phasematching condition. When the sample is larger than the beam spot, CARS emission mainly propagates forward. But, if the structure of the sample is smaller than the beam spot, not a little CARS emission propagates backward. Therefore, the component of the non-resonant background from the surrounding media, mainly water in the biological sample, is reduced by epi-direction. Since the polarization features of non-resonant background is different from that of CARS, the non-resonant background is removed with proper polarization angles of the $\omega_{1}$ and $\omega_{2}$ beams and an analyzer.
The combination of these methods, high spectral resolution, and the electric resonance to increase quantum efficiency will be a considerable breakthrough. We consider that the realtime imaging of non-staining molecule will be available in the near-future.

\section{Acknowledgement}

This work is the result of the collaboration with Prof. T. Araki (Osaka university) and Prof. S. Kawata (Osaka university). The author is grateful to them.

\section{References}

1. Cheng, J., Book, L. D. and Xie, X. S.: Polarization coherent antiStokes Raman scattering microscopy. Optics Lett. 26; 13411343, 2001.

2. Denk, W., Strickler, J. H. and Webb, W. W.: Two-photon laser scanning fluorescence microscopy. Science 248; 73-76, 1990.

3. Duncan, M. D., Reintjes, J. and Manuccia, T. J.: Scanning coherent anti-Stokes Raman microscope. Opt. Lett. 7; 350, 1982.

4. Hashimoto, M. and Araki, T.: Coherent anti-Stokes Raman scattering microscope. Proc. of SPIE 3749; 496-497, 1999.

5. Hashimoto, M. and Araki, T.: Three dimensional coherent and optical transfer functions of coherent anti-stokes Raman scattering microscopy. J. Opt. Soc. Am. A 18; 771-776, 2001.

6. Hashimoto, M., Araki, T. and Kawata, S.: Molecular vibration imaging in the fingerprint region by CARS Microscopy with collinear configuration. Opt. Lett. 25; 1768-1770, 2000.

7. Hashimoto, M., Araki, T. and Kawata, S.: Multi-focus coherent anti-Stokes Raman scattering microscopy. (in preparation)

8. Ichihara, A., Tanaami, T., Isozaki, K., Sugiyama, Y., Kosugi, Y., Kimuriya, K., Abe, M. and Umemura, I.: High-speed confocal fluorescence microscopy using a Nipkow scanner with microlenses for 3-D imaging of single fluorescent molecule in real time. Bioimages 4; 57-62, 1996.

9. Levenson, M. D., and Kano, S.: Introduction to Nonlinear Laser Spectroscopy, Academic press, Orlando, 1988.

10. Müller, M., Squier, J., de Lange, C. A. and Brakenhoff, G. J.: CARS microscopy with folded BoxCARS phasematching. $J$. Microsc. 197; 150-158, 2000.

11. Shen, Y. R.: The Principles of Nonlinear Optics, John Wiley Sons, New York, 1984.

12. Tiziani, H. J. and Uhde, H. M.: Three-dimensional analysis by microlens-array confocal arrangement. Appl. Opt. 33; 567-572, 1994.

13. Volkmer, A., Cheng, J. and Xie, X. S.: Vibrational imaging with high sensitivity via epidetected coherent anti-Stokes Raman scattering microscopy. Phys. Rev. Lett. 87; 023901-1-4, 2001.

14. Zumbusch, A., Holtom, G. R. and Xie, X. S.: Three-dimensional vibrational imaging by coherent anti-Stokes Raman scattering. Phys. Rev. Lett. 82; 4142-4145, 1999. 